\title{
Ventricular energetics after the Fontan operation: Contractility-afterload mismatch
}

\author{
Gábor Szabó, MD, $\mathrm{PhD}^{\mathrm{a}}$ \\ Volker Buhmann, MS \\ Andy Graf, MS \\ Sergei Melnitschuk, $M D^{b}$ \\ Susanne Bährle, $M^{\mathrm{C}}$ \\ Christian F. Vahl, MDa \\ Siegfried Hagl, MD ${ }^{a}$
}

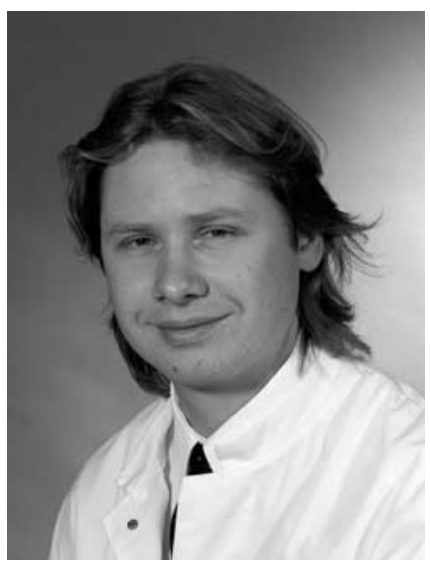

Dr Szabó

\footnotetext{
From the Department of Cardiac Surgery, University of Heidelberg, Germany ${ }^{\mathrm{a}}$; the Department of Cardiac Surgery, University of Zürich, Switzerland ${ }^{\mathrm{b}}$; and the Departments of Cardiology, Angiology and Pulmonology, University of Heidelberg, Germany. ${ }^{\mathrm{c}}$

Supported by grants "Forschungsschwerpunkt Transplantation," University of Heidelberg, and SFB414, German Research Foundation.

Read at the Eighty-second Annual Meeting of The American Association for Thoracic Surgery, Washington, DC, May 5-8, 2002.

Received for publication May 9, 2002; revisions requested May 30, 2002; revisions received June 19, 2002; accepted for publication July 12, 2002.

Address for reprints: Gábor Szabó, MD, $\mathrm{PhD}$, Department of Cardiac Surgery, Im Neuenheimer Feld 110, 69120 Heidelberg, Germany (E-mail: dzsi@hotmail.com).

J Thorac Cardiovasc Surg 2003;125:1061-9

Copyright (C) 2003 by The American Association for Thoracic Surgery

0022-5223/2003\$30.00+0

doi: $10.1067 / \mathrm{mtc} .2003 .405$
}

Objective: Fontan-type operations offer the opportunity to create pulmonary and systemic circulation in series with a single pumping chamber. The effectiveness of such a circulatory pattern determines resting and exercise hemodynamics in these patients. The present study investigated cardiac performance after the Fontan operation by using ventricular-vascular coupling framework analysis.

Methods: In 12 anesthetized open-chest dogs, Fontan circulation was established by using a cavopulmonary anastomosis. Left ventricular hemodynamic variables were measured by using a combined pressure-volume-conductance catheter. Additionally, aortic flow and pressure were recorded continuously. Ventricular contractility was quantified by using the load-independent slope of the endsystolic pressure-volume relationship. Arterial system properties were quantified by using the end-systolic pressure/stroke volume ratio. The coupling between the left ventricle and arterial system was expressed by using the ratio of end-systolic pressure/stroke volume to slope of the end-systolic pressure-volume relationship. Additionally, external stroke work, total mechanical energy and mechanical efficiency (Mechanical efficiency $=$ Stroke work/Total mechanical energy) were calculated. Impedance spectra were determined by means of Fourier analysis.

Results: During Fontan circulation, the slope of the end-systolic pressure-volume relationship $(5.3 \pm 0.6$ vs $7.5 \pm 0.6 \mathrm{~mm} \mathrm{Hg} / \mathrm{mL}, P<.05)$ decreased, and the end-systolic pressure-stroke volume relationship $(4.2 \pm 0.7$ vs $3.3 \pm 0.5 \mathrm{~mm}$ $\mathrm{Hg} / \mathrm{mL}, P=.23$ ) increased with parallel increased characteristic impedance. Furthermore, the end-systolic pressure-stroke volume/slope of the end-systolic pressure-volume relationship ratio increased significantly $(0.76 \pm 0.04$ vs $0.42 \pm 0.03$, $P<.005)$. Simultaneously, stroke work $(1846 \pm 146$ vs $1389 \pm 60 \mathrm{~mm} \mathrm{Hg} / \mathrm{mL}$, $P<.05)$ and mechanical efficiency $(0.82 \pm 0.09$ vs $0.56 \pm 0.05, P<.05)$ were significantly reduced.

Conclusions: Fontan circulation leads to contractility-afterload mismatch by means of increased impedance caused by additional connection of the pulmonary vascular bed to the systemic vasculature and by means of deterioration of myocardial contractility. The increased ventriculoarterial coupling ratio and reduced mechanical efficiency predict limited cardiac functional reserve after the Fontan operation. 


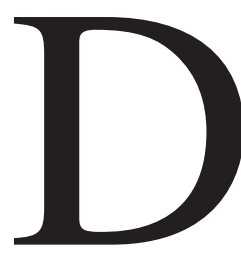

uring the past decades, different types of Fontan operations ${ }^{1,2}$ became the primary therapeutic option for physiologic correction of congenital heart disease in which repair into a 2 -ventricle system is impossible. In this situation the pulmonary and systemic circulations are in series with only one pumping chamber. Thus, the single ventricle must provide energy for pulmonary, as well as systemic, blood flow. Generally, patients who have undergone the Fontan operation have a good prognosis, although they have subnormal cardiac output $(\mathrm{CO})$ at rest, ${ }^{3,4}$ whereas central venous pressure is significantly increased. ${ }^{5}$ Despite sufficient resting hemodynamics, exercise performance is usually reduced, $, 3,4,6-8$ which thus far is attributed to cardiac factors, such as the absence of the right ventricle or impaired left ventricular function. However, because of the mechanical coupling between the heart and peripheral circulation, inadequate $\mathrm{CO}$ response to exercise might also be dependent on insufficient peripheral vascular adjustments.

To date, no experimental or clinical study provides a detailed analysis of ventriculoarterial mechanics after the Fontan operation. Although some theoretical models ${ }^{6,9}$ have been used to predict the interaction of different determinants of ventricular function, such as preload, afterload, and contractility, none of them has been validated. Nogaki and colleagues $^{9}$ postulated that in the Fontan circulation increased impedance and the lack of a compensatory increase in contractility might explain in part the abnormal functional status and decrease in survival after this procedure. For better understanding of ventricular mechanoenergetics, we assessed ventricular and vascular properties by means of pressure-volume and impedance spectrum analysis in an experimental model of Fontan circulation.

\section{Materials and Methods \\ Animals}

Twelve dogs (foxhounds) weighing 16 to $32 \mathrm{~kg}(22.3 \pm 5.2 \mathrm{~kg})$ were used in this experiment. All animals received humane care in compliance with the "Principles of Laboratory Animal Care" formulated by the National Society for Medical Research and the "Guide for the Care and Use of Laboratory Animals" prepared by the Institute of Laboratory Animal Resources and published by the National Institutes of Health (National Institutes of Health Publication no. 86-23, revised 1996).

\section{Surgical Preparation and Experimental Design}

General management. The dogs were premedicated with propionylpromazine and anesthetized with a bolus of pentobarbital (15 mg/kg initial bolus, followed by $0.5 \mathrm{mg} \times \mathrm{kg}^{-1} \times \mathrm{h}^{-1}$ administered intravenously), paralyzed with pancuronium bromide $\left(0.1 \mathrm{mg} / \mathrm{kg}\right.$ as a bolus, followed by $0.2 \mathrm{mg} \times \mathrm{kg}^{-1} \times \mathrm{h}^{-1}$ administered intravenously), and endotracheally intubated. The dogs were ventilated with a mixture of room air and oxygen (fraction of inspired oxygen $=60 \%$ ) at a frequency of 12 to 15 breaths/min and a tidal volume starting at $15 \mathrm{~mL} \times \mathrm{kg}^{-1} \times$ $\min ^{-1}$. The settings were adjusted by maintaining arterial partial carbon dioxide pressure between 35 and $40 \mathrm{~mm} \mathrm{Hg}$. The femoral artery and vein were cannulated for recording aortic pressure (AoP) and taking blood samples for the analysis of blood gases, electrolytes, and $\mathrm{pH}$. Basic intravenous volume substitution was carried out with Ringer solution at a rate of $1 \mathrm{~mL} \times \min ^{-1} \times$ $\mathrm{kg}^{-1}$. According to the values of potassium, bicarbonate, and base excess, substitution included administration of potassium chloride and sodium bicarbonate $(8.4 \%)$. Neither catecholamines nor other hormonal or pressor substances were administered. Rectal temperatures and standard peripheral electrocardiographic results were monitored continuously.

Fontan circulation. After lateral thoracotomy in the fifth intercostal space and pericardiotomy, the great vessels were dissected and isolated, and the azygos vein was ligated. The Fontan circulation was established according to previous models (Figure 1). ${ }^{10,11}$ Two $16 \mathrm{~F}$ polyethylene cannulas were introduced into the superior and inferior venae cavae, and a third $18 \mathrm{~F}$ cannula was introduced into the distal main pulmonary trunk. They were connected to a Y-shaped connector with a 3-way stopcock for deairing. Then the venae cavae (superior and inferior) were closed with tourniquets at the level of the cannula in a stepwise fashion, and the blood was directed from the systemic venous system into the main pulmonary artery, bypassing the right side of the heart. A $12 \mathrm{~F}$ vent was introduced into the right ventricle to collect coronary venous blood, which was directed into the external jugular vein with a small roller pump.

\section{Data Acquisition and Analysis}

AoP, pulmonary arterial pressure (PAP), right atrial pressure (RAP), and left atrial pressure (LAP) were monitored with $5 \mathrm{~F}$ Millar catheter-tip manometers (Millar Instruments, Inc, Houston, Tex). Aortic and pulmonary artery flow were measured with perivascular electromagnetic flowmeters. A combined $6 \mathrm{~F}$ Millar pressure-conductance catheter with 6-mm spacing was inserted into the left ventricle through the apex to collect left ventricular pressure-volume data. Left ventricular systolic pressure, maximum pressure development, left ventricular end-diastolic pressure, and $\mathrm{CO}$ as the equivalent of aortic flow were monitored continuously. Stroke volume (SV) was calculated from the integrated flow signal and was used to calibrate the volume signal from the conductance catheter. The volume signal provided by the conductance catheter was registered continuously (Sigma F5; Leycom, Leiden, The Netherlands) and computed by using the Conduct PC software (Leycom, Leiden, The Netherlands). Pressure-volume loops were constructed online. Vena caval occlusions were performed to obtain a series of loops for calculation of the slope (Ees) and intercept (V0) of the end-systolic pressure-volume relationship according to the following equation:

$$
\text { Pes }=\text { Ees }(\text { Ves }- \text { V0). }
$$

Arterial elastance (Ea) was calculated as follows:

$$
\mathrm{Ea}=\mathrm{Pes} / \mathrm{SV} \text {. }
$$

Ventriculoarterial coupling was described by the quotient of Ea and Ees. ${ }^{12}$ According to Sunagawa and associates, ${ }^{12}$ stroke work (SW) was calculated as the area within the pressure-volume loop, 
TABLE 1. Hemodynamic parameters

\begin{tabular}{lcc}
\hline & Baseline & Fontan \\
\hline HR (min) & $109 \pm 6$ & $111 \pm 5$ \\
MAP (mm Hg) & $86 \pm 7$ & $92 \pm 8$ \\
CO (mL/min) & $2766 \pm 211$ & $2157 \pm 308$ \\
SV (mL) & $25.5 \pm 2.1$ & $20.0 \pm 1.6^{*}$ \\
ESP $(\mathrm{mm} \mathrm{Hg})$ & $75 \pm 6$ & $73 \pm 6$ \\
ESV $(\mathrm{mL})$ & $14.0 \pm 2.0$ & $14.7 \pm 1.5$ \\
EDP $(\mathrm{mm} \mathrm{Hg})$ & $9.0 \pm 1.7$ & $6.7 \pm 1.1$ \\
EDV $(\mathrm{mL})$ & $39.5 \pm 2.7$ & $33.7 \pm 3.5^{*}$ \\
PAP $(\mathrm{mm} \mathrm{Hg})$ & $9.3 \pm 2.1$ & $20.2 \pm 5.3^{*}$ \\
SVR (dynes $\left.\cdot \mathrm{s}^{-1} \cdot \mathrm{cm}^{-5}\right)$ & $2458 \pm 350$ & $2670 \pm 340$ \\
PVR (dynes $\left.\cdot \mathrm{s}^{-1} \cdot \mathrm{cm}^{-5}\right)$ & $144 \pm 27$ & $556 \pm 43^{*}$ \\
TVR (dynes $\left.\cdot \mathrm{s}^{-1} \cdot \mathrm{cm}^{-5}\right)$ & - & $3226 \pm 220 \dagger$ \\
\hline
\end{tabular}

All values are given as means \pm SEM. $H R$, Heart rate; $M A P$, mean arterial pressure; $E S P$, end-systolic pressure; ESV, end-systolic volume; EDP, end-diastolic pressure; $E D V$, end-diastolic volume; $T V R$, total vascular resistance.

${ }^{*} P<.05$, Fontan versus baseline.

$\dagger P<.05$, Fontan total vascular resistance versus baseline SVR.

and pressure-volume area (PVA) was calculated as the area circumscribed by the end-systolic pressure-volume line, the enddiastolic pressure-volume relation curve, and the systolic pressurevolume trajectory. The SW/PVA ratio was defined as mechanical efficiency. ${ }^{12}$

Because flow through the cardiovascular system is pulsatile, conventional analysis excludes the significant contribution of pulsatile flow to the understanding of systemic hemodynamics. This leads to an underestimation of ventricular energy requirements. Therefore, we calculated the vascular impedance spectrum through Fourier transformation. ${ }^{13,14}$ The concept of Fourier analysis is based on the general principle that periodic waves can be mathematically expressed as a sum of a series of pure sinusoidal harmonics. This Fourier series contains a zero frequency (mean) and oscillatory harmonic frequencies that are integer multiples of the original periodic wave form. At each individual harmonic, division of the pressure amplitude by the flow amplitude allowed calculation of the oscillatory counterpart of resistance, or impedance, at that respective harmonic. Input impedance was the impedance calculated at the zero harmonic and is a measure of resistance to mean systemic blood flow. Characteristic impedance was estimated as the mean impedance between 2 and $12 \mathrm{~Hz}$ and is a measure of resistance to pulsatile blood flow.

In addition, systemic vascular resistance (SVR) was calculated as follows:

$$
\mathrm{SVR}=(\mathrm{AoP}-\mathrm{RAP}) / \mathrm{CO}
$$

and pulmonary vascular resistance (PVR) was calculated as follows:

$$
\mathrm{PVR}=(\mathrm{PAP}-\mathrm{LAP}) / \mathrm{CO}
$$

Total vascular resistance of the single ventricle in the Fontan circulation was calculated as the sum of SVR and PVR, whereas RAP was substituted by PAP at the calculation of SVR.

All hemodynamic parameters were registered on a Gould multichannel monitor unit (Gould Instrument Systems, Valley View, Ohio) and recorded on a personal computer for further offline analysis. Hemodynamic measurements were performed before and 60 minutes after completion of Fontan circulation.

\section{Statistics}

Statistical analysis was performed on a personal computer with commercially available software (Origin 5.0; OriginLab, Northampton, Mass). All values were expressed as means \pm SEM. Hemodynamic data taken before and after Fontan circulation were analyzed with standard 2-tailed paired Student $t$ tests.

\section{Results}

Hemodynamic variables are shown in Table 1. Baseline values were within the physiologic range. After completion of the Fontan circulation, a minimal pressure gradient of $0.85 \pm 0.42 \mathrm{~mm} \mathrm{Hg}$ was observed over the cavopulmonary connection. Most of the hemodynamic parameters showed only marginal changes. Heart rate, mean AoP, end-systolic pressure, and end-systolic volume were nearly identical to baseline values. In contrast, end-diastolic volume and SV decreased significantly $(P<.05$, Figure 2$)$. CO showed only a strong decreasing tendency, without reaching the level of significance. PAP increased significantly $(P<.05)$. Although SVR showed only a slight increase, PVR increased significantly $(P<.05)$. Total vascular resistance (calculated as SVR plus PVR) of the single ventricle was significantly $(P<.05)$ higher than the SVR of the left ventricle in biventricular circulation.

Figure 1 shows representative series of pressure-volume loops. The end-systolic pressure-volume trajectory became flatter $\left(\operatorname{Ees}_{\mathrm{f}} \mathrm{vs}\right.$ Ees $)$ and the arterial elastance curve $\left(\mathrm{Ea}_{\mathrm{f}} \mathrm{vs}\right.$ Ea) became steeper after Fontan circulation. Pressure-volume analysis revealed a significant decrease of Ees $(P<$ .05 ) and an increase of Ea, without reaching the level of significance (Figure 3). Subsequently, the ventriculoarterial coupling ratio $(\mathrm{Ea} / \mathrm{Ees})$ dramatically increased $(P<.005)$. 


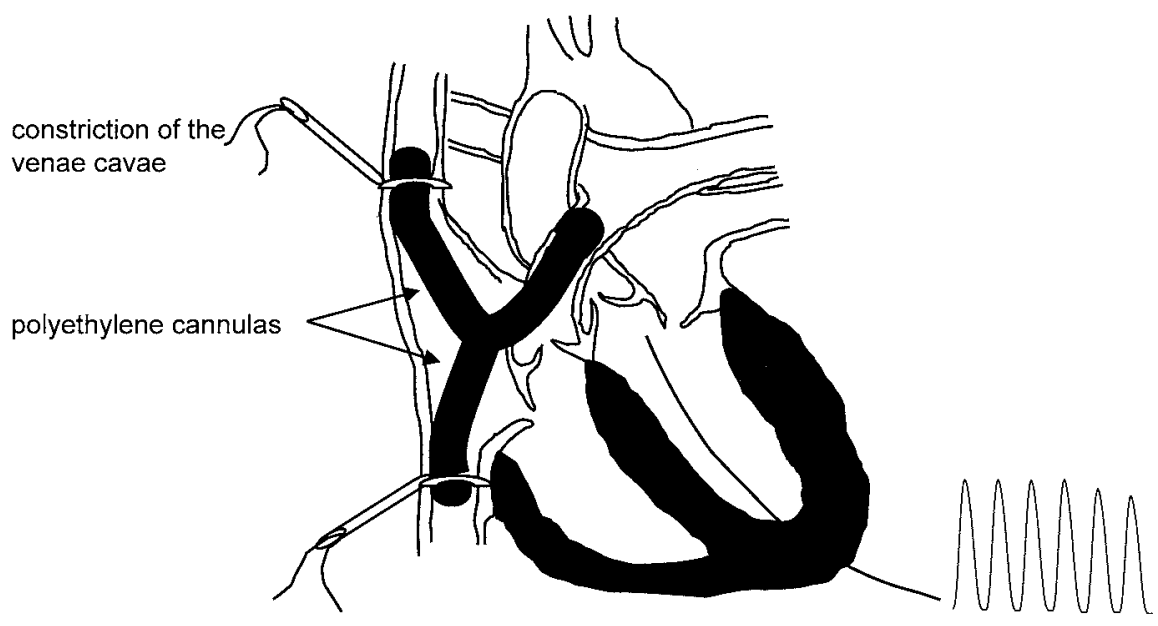

LV pressure-conductance catheter

Figure 1. Schematic representation of the experimental model. Univentricular circulation was created with polyethylene cannulas directing the blood from the venae cavae directly into the main pulmonary artery (cavopulmonary bypass). LV, Left ventricular.

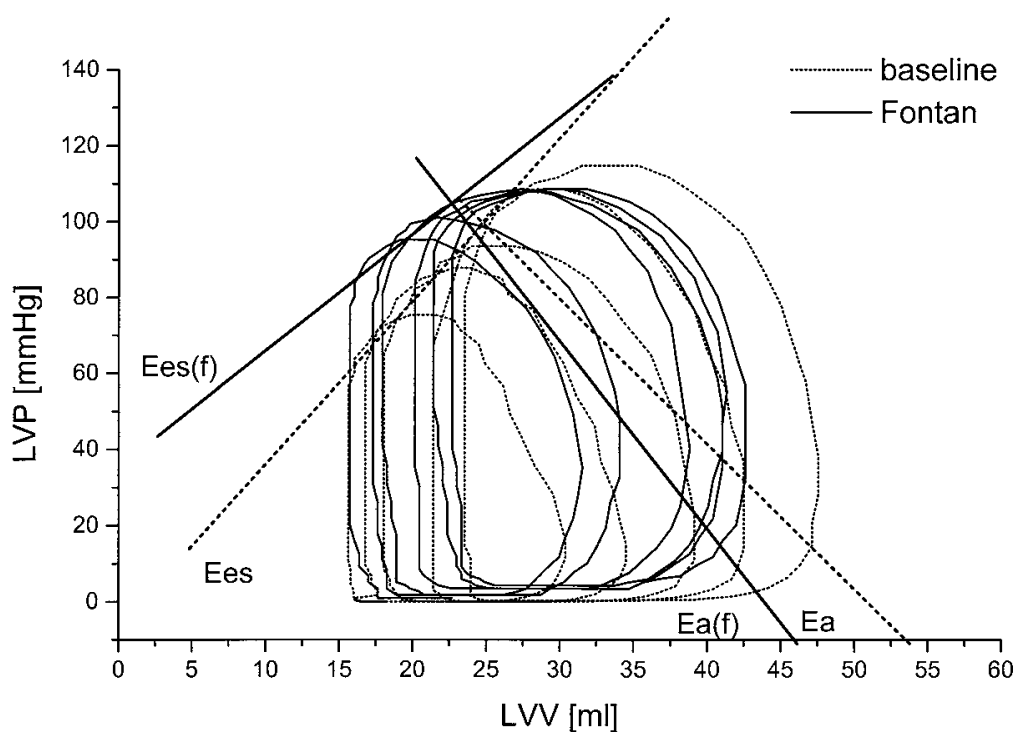

Figure 2. Representative series of pressure-volume loops before (dotted line) and after (solid line, $f$ in index) Fontan operation. The end-systolic pressure-volume ( $\mathrm{Ees}_{\mathrm{f}}$ vs $\left.\mathrm{Ees}\right)$ and arterial elastance $\left(\mathrm{Ea}_{\mathrm{f}} \mathrm{vs} \mathrm{Ea}\right)$ trajectories are indicated. LVP, Left ventricular pressure; LVV, left ventricular volume.

Similarly, SW decreased significantly $(P<.05)$, and PVA remained unchanged, resulting in a markedly reduced mechanical efficiency $(P<.05$, Figure 4$)$.

Fourier analysis of impedance spectrums (Figure 5) showed an increase of input impedance, without reaching the level of significance. Characteristic impedance increased significantly $(P<.05)$ after the Fontan operation (Figure 5).

\section{Discussion}

In the clinical situation recipients of Fontan circulation exhibit quite normal systemic arterial pressure, a moderate reduction in $\mathrm{CO}$ and $\mathrm{SV}$, and an increased venous pressure. ${ }^{3-5,7,8}$ This indicates that after the Fontan operation, the single ventricle is able to maintain a sufficient circulation at rest, which becomes critical in response to exercise in comparison with the normal circulation. This impairment is 

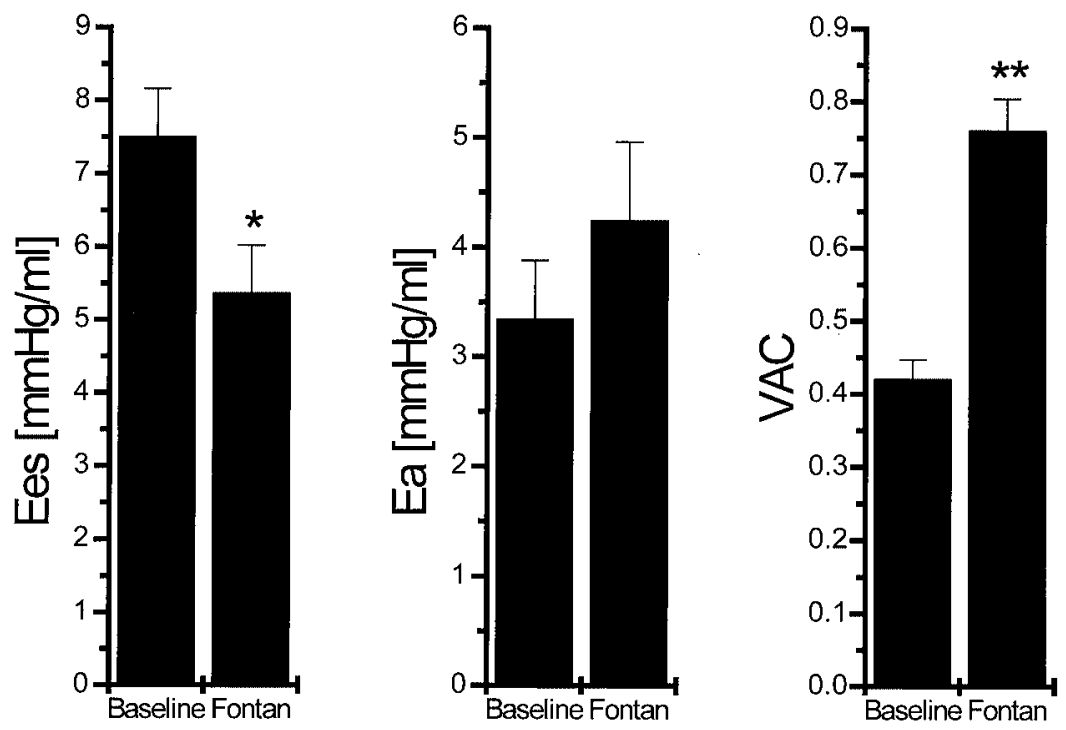

Figure 3. Changes of Ees (left panel), Ea (middle panel), and ventriculoarterial coupling (VAC = Ea/Ees, right pane). All values are given as means \pm SEM: ${ }^{*} \boldsymbol{P}<.05$, Fontan versus baseline; ${ }^{*} \boldsymbol{P}<.005$, Fontan versus baseline.
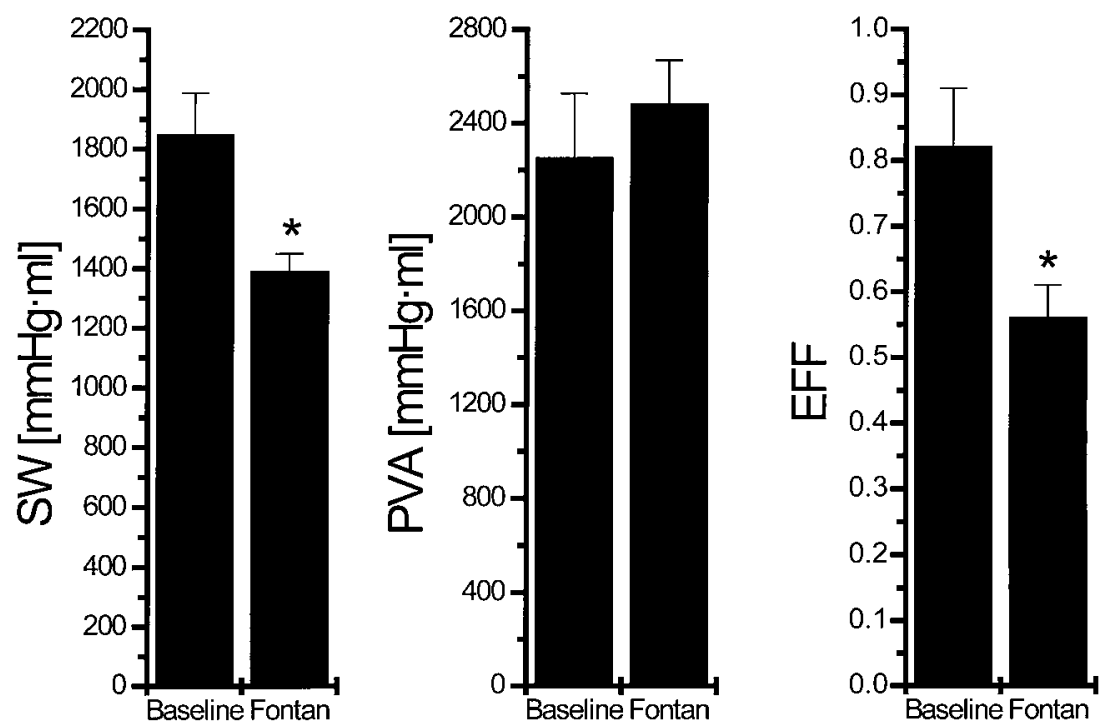

Figure 4. Changes of SW (left panel), PVA (middle panel), and mechanical efficiency (EFF, right panel). All values are given as means \pm SEM: ${ }^{*} P<.05$, Fontan versus baseline.

mainly evident in the insufficient capacity to increase SV and hence $\mathrm{CO}$ during exercise. ${ }^{3,4,6-8}$ Although these aspects are well documented, a clear understanding of the phenomena involved and the key role of individual hemodynamic factors is still lacking.

Therefore, we used pressure-volume and impedance spectrum analysis to characterize mechanoenergetic changes in the univentricular circulation. We determined Ees and Ea, which are relative load-independent indices of ventricular contractility and vascular loading, respective- ly. ${ }^{12}$ Although the changes of several basic hemodynamic parameters were rather small, these changes had a profound influence on mechanoenergetics. The parallel decrease of myocardial contractility (Ees) and the slight increase of Ea led to a highly significant worsening of the ventriculoarterial coupling ratio. Similarly, the decrease of SW combined with the unchanged pressure-volume area (total mechanical energy) resulted in a significant reduction of mechanical efficiency. Our findings confirm those in the study of Tanoue and colleagues, ${ }^{15}$ in which the ventriculoarterial cou- 

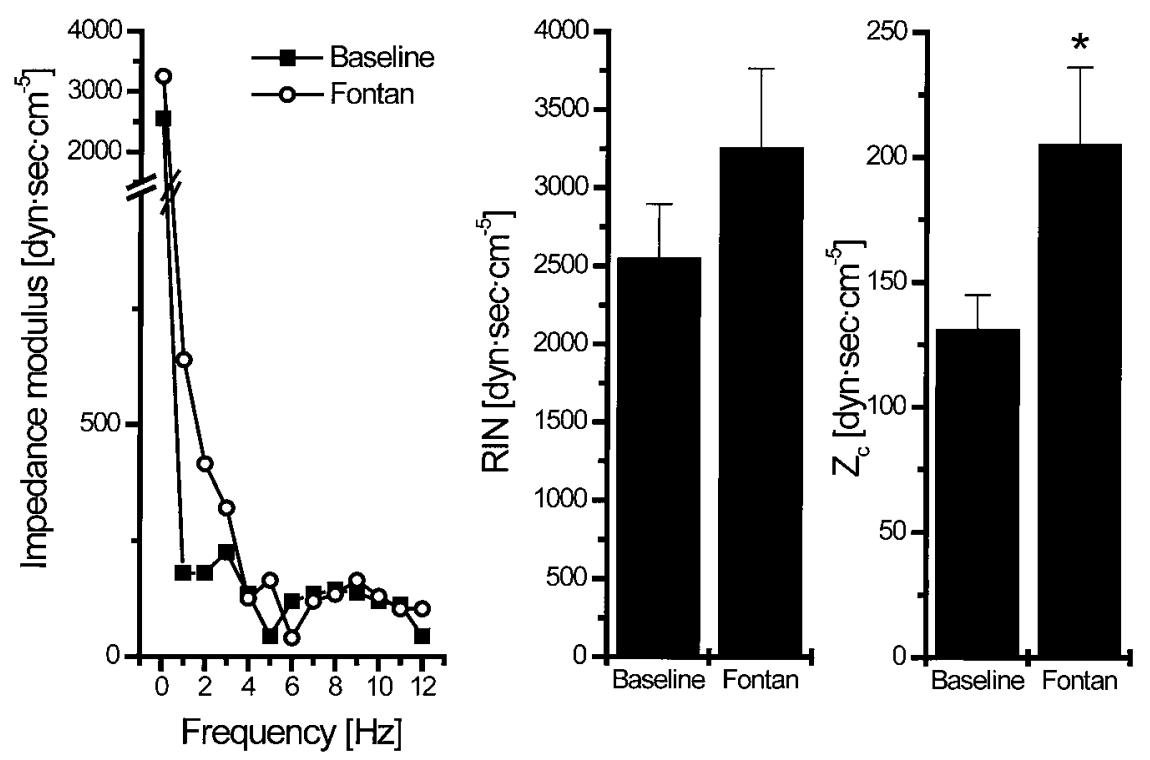

Figure 5. Impedance spectrum (left panel), input impedance (middle panel), and characteristic impedance $\left(Z_{c^{\prime}}\right.$ right pane $)$ are shown. All values are given as means \pm SEM: ${ }^{*} P<.05$, Fontan versus baseline.

pling ratio was approximated from routine cardiac catheterization data in patients undergoing clinical Fontan procedures. They found a worsened ventriculoarterial coupling (increased Ea/Ees) and thereby contractility-afterload mismatch after primary total cavopulmonary connection. In accordance with previous theoretical studies, ${ }^{6,9}$ the results of the present study indicate that to maintain physiologic conditions, the single ventricle in the Fontan circulation has to provide almost maximum power with low efficiency. In contrast, to maintain physiologic conditions, a left ventricle in the normal circulation can provide a higher SW with a higher efficiency than in Fontan circulation. In these aspects, the adaptability of Fontan circulation to conditions in which high ventricular energy is required appears to be limited.

The data of the present study suggest that multiple (and in certain cases small) changes of afterload, preload, and contractility additively result in unfavorable mechanoenergetics in the Fontan circulation. We determined ventricular afterload in terms of vascular resistance and arterial elastance, as well as input impedance and characteristic impedance. SVR per se did not change significantly, which is consistent with the study of Macé and coworkers, ${ }^{11}$ who used a nearly identical model of Fontan circulation in pigs. However, the series of SVR and PVR represented a significantly higher afterload for the single ventricle in the Fontan circulation than SVR alone for the left ventricle in the normal circulation. ${ }^{6,9}$ The strong tendency $(P=.08)$ toward increased arterial elastance in the Fontan circulation confirms previous theoretical assumptions ${ }^{6}$ and also suggests a moderate increase of afterload. To further elucidate after- load changes, we analyzed impedance spectrums. Although the increase of input impedance (zero harmonics, an equivalent of vascular resistance) did not reach the level of significance, characteristic impedance was significantly increased. This indicates an increased stiffness of the arterial tree. ${ }^{16}$ The causes of increased characteristic impedance remain unclear. However, previous studies suggest ${ }^{5,6}$ that sympathetic activation as a compensatory mechanism to maintain arterial pressure at a reduced $\mathrm{CO}$ might play a central role. Indeed, Kelley and associates ${ }^{5}$ found increased forearm vascular resistance, increased heart rate, and increased resting plasma norepinephrine levels in patients undergoing clinical Fontan procedures in comparison with values in healthy control subjects.

A very important finding of the present study is that the single ventricle inadequately uses the heterometric (FrankStarling mechanism) and homeometric (Anrepp effect) autoregulation in face of increased afterload in the Fontan circulation: instead of a compensatory increase of preload, contractility, or both, both determinants of ventricular function are reduced. In our study preload reduction could be characterized by the significant decrease of end-diastolic volume. The main reason might be the reduced venous return in the univentricular circulation associated with the increased central venous-main PAPs, which are essential to maintain pulmonary circulation. Macé and coworkers ${ }^{11}$ investigated the changes of venous return parameters by means of Guytonian (mean filling pressure-systemic blood flow) relationships. They found that in the univentricular circulation the slope of the Guytonian relationship significantly decreases, and the pressure intercept at zero venous 
return significantly increases. They concluded that placing SVR and PVR in series induces a decrease in the slope of the Guytonian relationship. The greater the decrease in the slope, the greater increase in the driving force or pressure gradient for venous return is necessary to keep $\mathrm{CO}$ constant. This also confirms the data of Lee and colleagues, ${ }^{17}$ which show in conscious normal dogs that preload-afterload mismatch might occur if the afterload increases but the venous return is inadequate to maintain $\mathrm{SV}$.

In addition to reduced venous return, the above-described alterations of characteristic impedance itself might influence ventricular preload. Yano and coworkers ${ }^{16}$ reported that an isolated increase of characteristic impedance caused by stiffening the aorta with a perivascular rigid tube leads to a decrease in preload and SV and thereby to a preload-afterload mismatch in the intact heart. Berger and associates ${ }^{18}$ systematically investigated the determinants of wave propagation in the coupled left ventricle-arterial system. They described a linear inverse relationship between characteristic impedance and SV: an isolated increase in characteristic impedance led to a subsequent decrease in $\mathrm{SV}$. This indicates that the isolated increase of characteristic impedance might also contribute to decreased preload and $\mathrm{SV}$ in the Fontan circulation.

As a third determinant of ventricular function, myocardial contractility decreased. To the best our best knowledge, this is the first study that describes contractile function by means of end-systolic pressure-volume relationships in Fontan circulation. The observed changes of afterload and preload might have opposite effects on contractility. Many authors ${ }^{19-21}$ described that increased afterload leads to an increased contractility, even in the absence of neural control. In contrast, the decrease of preload leads to a decrease of myocardial contractility through reduced affinity of contractile proteins for calcium, altered intracellular calcium release, action potential changes, and stretch-activated ion channels. ${ }^{22}$ Because the increase of systemic afterload was rather small and preload reduction seemed to be more prominent, reduced contractility probably reflects the net effect of both preload and afterload changes. Also, clinical studies $^{23}$ confirm reduced contractile function and altered loading conditions after Fontan procedures. The intracellular mechanisms leading to reduced contractility require further investigation.

There are some limitations of the present study. We performed Fontan operations in healthy animals with normal preoperative mechanoenergetics. In contrast, in the clinical situation the preoperative patient undergoing the Fontan operation has a chronic volume overload that leads to congestive heart failure over a longer time period. Volume unloading after the Fontan procedure is associated with a decrease in end-systolic stress and increase in contractility ${ }^{24}$ On the other hand, chronic volume overload might induce changes in myocardial structure, which limit the improvement of contractile function. ${ }^{24}$ Furthermore, the type of underlying cardiac disease, single-ventricle morphology (left vs right), previous cardiac history, chronic hypoxemia, and extent of pulmonary hypertension, as well as the type of Fontan repair, might also influence the postoperative hemodynamics. ${ }^{15,24}$ Tanoue and colleagues ${ }^{15}$ showed that volume reduction by means of bidirectional Glenn anastomosis preceding total cavopulmonary connection improves afterload mismatch and thereby improves ventricular energetics after total cavopulmonary connection. In the present study we observed acute changes directly after conversion into the univentricular circulation. However, a long-term adaptation to the Fontan circulation might induce other compensatory mechanisms that cannot be ruled out in our acute experiments.

In this context it has to be mentioned that we observed a marked increase in PVR consistently with previous experimental data in cavopulmonary connection, ${ }^{10,11}$ as well as in atriopulmonary connection, ${ }^{25,26}$ which does not necessarily occur in chronic Fontan patients. This point remains unclear because only very few clinical studies determined PVR in a limited number of patients, and none of these studies reported reference values in a healthy control population. Serraf and colleagues ${ }^{27}$ described a significant increase in the quotient of pulmonary-to-systemic resistance after the Fontan procedure in comparison with preoperative values. Summarizing the available data, PVR is increased during the early postoperative period as a result of the release of vasoactive substances, such as endothelin $1 .{ }^{28} \mathrm{Here}$, further studies are warranted to investigate the regulation of pulmonary vasculature in the Fontan circulation. In addition, a model study of Tamaki and coworkers ${ }^{29}$ indicates that nonpulsatile flow might also contribute to an increased PVR after the Fontan procedure. These aspects must be taken into account when interpreting the data.

In summary, we showed that even small but complex changes of preload, afterload, and myocardial contractility in the Fontan circulation have profound effects on ventriculoarterial mechanoenergetics in terms of contractility-afterload mismatch and reduced mechanical efficiency. These alterations might also limit the adaptation potential of the single ventricle during exercise. For better understanding of the clinical situation, future studies are needed on the effects of Fontan circulation in chronically volume-overloaded hearts.

\section{References}

1. Fontan F, Baudet E. Surgical repair of tricuspid atresia. Thorax. 1971;26:240-8.

2. de Leval MR, Kilner P, Gewillig M, Bull C, McGoon DC. Total cavopulmonary connection: a logical alternative to atriopulmonary connection for complex Fontan operations. J Thorac Cardiovasc Surg. 1988;96:682-95. 
3. Schachar GB, Fuhrman BP, Wang Y, Lucas RV, Lock JE. Rest and exercise hemodynamics after Fontan procedure. Circulation. 1982;65: 1043-8.

4. Zellers TM, Driscoll DJ, Mottram CD, Puga FJ, Hartzell VS, Danielson GK. Exercise tolerance and cardiorespiratory response to exercise before and after Fontan operation. Mayo Clin Proc. 1989;64:1489-97.

5. Kelley JR, Mack GW, Fahey JT. Diminished venous capacitance in patients with univentricular hearts after the Fontan operation. Am J Cardiol. 1995;76:158-63.

6. Magosso E, Cavalcanti S, Ursino M. Theoretical analysis of rest and exercise hemodynamics with total cavopulmonary connection. Am J Physiol. 2002;282:H1018-34.

7. Akagi T, Benson LN, Green M, De Souza M, Harder JR, Gilday DL, et al. Ventricular function during supine bicycle exercise in univentricular connection with absent right atrioventricular connection. Am J Cardiol. 1991;67:1273-8.

8. Rosenthal M, Bush A, Deanfield J, Redington A. Comparison of cardiopulmonary adaptation during exercise in children after the atriopulmonary and total cavopulmonary connection Fontan procedures. Circulation. 1995;91:372-8.

9. Nogaki M, Senzaki H, Masutani S, Kobayashi J, Kobayashi T, Sasaki $\mathrm{N}$, et al. Ventricular energetics in Fontan circulation: Evaluation with a theoretical model. Pediatr Int. 2000;42:651-7.

10. Nawa S, Irie H, Takata K, Sugawara E, Teramoto S. Development of a new experimental model for total exclusion of the right heart without the aid of cardiopulmonary bypass. J Thorac Cardiovasc Surg. 1989; 97:130-4.

11. Macé L, Dervanian P, Bourriez A, Mazmanian GM, Lambert V, Losay $\mathrm{J}$, et al. Changes in venous return parameters associated with univentricular Fontan circulations. Am J Physiol. 2000;279:H2335-43.

12. Sunagawa K, Maughan D, Burkhoff D, Sagawa K. Left ventricular interaction with arterial load studied in isolated canine ventricle. Am J Physiol. 1983;254:H773-80.

13. O'Rourke M, Taylor MG. Input impedance of the systemic circulation. Circ Res. 1967;19:365-80.

14. Attinger EO, Anne A, McDonald DA. Use of Fourier series for the analysis of biological systems. Biophys J. 1996;6:291-304.

15. Tanoue Y, Sese A, Ueno Y, Joh K, Hijii T. Bidirectional Glenn procedure improves the mechanical efficiency of a total cavopulmonary connection in high-risk Fontan candidates. Circulation. 2001; 103:2176-80.

16. Yano M, Kohno M, Konishi M, Obayshi M, Kobayashi S, Seki K, et al. Effect of aortic impedance on preload-afterload mismatch in canine hearts in situ. Basic Res Cardiol. 1997;92:115-22.

17. Lee J, Tajimi T, Patritti J, Ross J. Preload reserve and mechanism of afterlaod mismatch in normal conscious dogs. Am J Physiol. 1986; 250:464-73.

18. Berger DS, Robinson KA, Shroff SG. Wave propagation in coupled left ventricle-arterial system. Implications for aortic pressure. Hypertension. 1996;27:1079-89.

19. Suga H, Sagawa K, Kostiuk DP. Controls of ventricular contractility assessed by pressure-volume ratio, $\mathrm{E}_{\max }$. Cardiovasc Res. 1976;10: 582-92.

20. Klautz RJM, Teitel DF, Steendijk P, Van Bel F, Baan J. Interaction between afterload and contractility in the newborn heart: evidence of homeometric autoregulation in the intact circulation. J Am Coll Cardiol. 1995;25:1428-35.

21. Asanoi H, Ishizaka S, Kameyama T, Sasayama S. Neural modulation of ventriculoarterial coupling in conscious dogs. Am J Physiol. 1994; 266:H741-8

22. Crozatier B. Stretch-induced modifications of myocardial performance: from ventricular function to cellular and molecular mechanisms. Cardiovac Res. 1996;32:25-37.

23. Akagi T, Benson LN, Green M, Ash J, Gilday DL, Williams WG, et al. Ventricular performance before and after Fontan repair for univentricular atrioventricular connection: angiographic and radionuclide assessment. J Am Coll Cardiol. 1992;20:920-6.

24. Sluysmans T, Sanders SP, Van der Velde M, Matitiau A, Parness IA, Spevak PJ, et al. Natural history and patterns of recovery of contractile function in single left ventricle after Fontan operation. Circulation. 1992;86:1753-61.
25. Brutel de la Riviere A, Haasler G, Malm JR, Bregman D. Mechanical assistance of the pulmonary circulation after right ventricular exclusion. J Thorac Cardiovasc Surg. 1983;6:809-14.

26. Haneda K, Konnai T, Sato N, Nicoloff NN, Mohri H. Acute hemodynamic changes after Fontan operation: an experimental study. Tohoku J Exp Med. 1993;169:113-9.

27. Serraf A, Houyel L, Nicolas F, Lacour-Gayet F, Bruniaux J, Petit J, et al. Pulmonary circulation evaluation before cavopulmonary connections: the cavopulmonary bypass. Ann Thorac Surg. 1994;58:1096102.

28. Hiramatsu T, Imai Y, Kurosawa H, Takanashi Y, Aoki M, Shinoka T, Nakazawa M. Effects of dilutional and modified ultrafiltration in plasma endothelin-1 and pulmonary vascular resistance after the Fontan procedure. Ann Thorac Surg. 2002;73:861-5.

29. Tamaki S, Kawazoe K, Yagihara T, Abe T. A model to simulate the hemodynamic effects of right heart pulsatile flow after modified Fontan procedure. Br Heart J. 1992;67:177-9.

\section{Discussion}

Mr Marc de Leval (London, United Kingdom). Dr Szabó and his colleagues must be congratulated on an important experimental contribution to our understanding of the ventricular energetics of the Fontan circulation.

This article is, to my knowledge, the first animal study of ventricular contractility and ventricular vascular coupling using load-independent indices derived from pressure-volume load of the Fontan circulation. You demonstrated that the Fontan heart faces an increased afterload and cannot use the compensatory mechanisms of the normal biventricular heart, which consist of an increased preload and contractility. You are confirming in an animal model what had been postulated from theoretical models. This, of course, gives some weight to your study.

I have a number of comments and a few questions.

You do not describe in detail your experimental model, but you refer to the work of Nawa and colleagues and to the very nice study published a few years ago by Macé and colleagues. I would challenge your assumption that those are valid models of the Fontan state. All these experiments, including yours, displayed the typical features of a failing Fontan circulation. There is a significant increase in PAP in all these studies, whereas in the good Fontan circulation, the PAP is at best the same or lower than the normal PAP.

In Nawa's article it is stated, and I quote, that "severe circulatory failure progressed after the mean PA pressure was reduced to 20 mm Hg." In Macé's original article, published in 1995, it is mentioned, and I quote again, that ". . the mean LAP [left atrial pressure] had to be significantly increased to keep the CI [cardiac index] constant." All these studies are acute and imply that animals could not survive. Therefore, my first question is to ask you to comment on the validity of your model as being a model of the Fontan state rather than the failing Fontan procedure.

You do not describe the method you used to decompress the right ventricle. I wonder whether some of the recorded changes could be related to ventricular interference between the right and left ventricles. For example, could a septal shift account for the reduction of the end-diastolic volume? This is my second question.

You do not give the results of the LAP, although you say in the "Methods" section that you measure it. I assume that it increases as it does in the experimental studies to which you refer. How do you explain the reduction in end-diastolic volume if there is an 
increasing LAP produced by an increased preload? This is my third question.

The increased afterload is obviously very complex, and you have shown very nicely the importance of characteristic impedance. The most striking feature in the hemodynamic changes is the dramatic increase in PVR. I have the impression that your impedance measurements are related to the systemic arterial system rather than the pulmonary arterial system. Could you clarify this for my own understanding of your measurements?

An increase in the systemic afterload has been reported in the clinical setting, and it has been recommended to treat those patients with afterload reducers. Is it logical to use afterload reducers, acting essentially on the systemic circulation, when the increased afterload is mainly due to increased PVR? Would pulmonary vasodilators, for example, be more appropriate than systemic vessel dilators? This is my next question.

Last, you postulate that the limited preload reserve is partly responsible for the reduction in exercise tolerance of the patients undergoing the Fontan operation. Do you think that an augmentation of ventricular preload through a fenestration could be recommended not only acutely but also chronically to improve exercise tolerance and therefore that these fenestrations should not be closed? This is my last question.

Dr Szabó. Thank you very much for your comments and questions.

Regarding the model, when I started this experimental series, I wondered why so little experimental research had been done using the Fontan circulation, and I had to determine that it is very hard to establish an acute Fontan circulation in normal hearts. This might have a reason in the fact that in the clinical situation the hearts are univentricular already before the Fontan operation and might be adapted to this special circulatory situation. It is clear that the model that we used and that was used in other articles before is, at this point, different from the clinical situation. However, despite this limitation, the model features the main important hemodynamic parameters regarding ventricular and arterial me- chanics and mimics the clinical situation in terms of normal arterial pressure, reduced $\mathrm{CO}$, and increased central venous pressure.

The decompression of the right ventricle was not shown in the graphs. We placed a small vent into the right ventricle so that the right ventricle was completely empty during the experiments, and the blood, the coronary blood, was reinfused into the animal by using a small roller pump.

The next question concerned the preload reduction. It was not the scope of our study to analyze how and why preload reduction occurs in this model. However, a very nice study from Magosso predicted, in a theoretical model, the significant reduction of the end-diastolic volume. The study of Macé showed, in terms of Guytonian relationships, that reduction of venous return is also a contributing factor to reduced preload. In addition, there is an article published in 1996 in Hypertension that describes the effects of an isolated increase of arterial afterload on preload. Berger and colleagues showed that an isolated increase of characteristic impedance leads to a reduction of SV and end-diastolic volume. They demonstrated an inverse linear correlation between characteristic impedance and preload. Therefore, this might also be an additional factor in terms of preload reduction.

The next question was whether the calculated impedance included PVR or only SVR. In the present study we calculated the impedance spectrum from aortic flow and pressure signals. It means that the impedances were calculated from the systemic part of the circulation, and the pulmonary part was not included.

This might also be an answer for the next question if afterload reduction therapy is an option in these patients. On the basis of the experimental data showing increased systemic vascular impedance, afterload-reducing therapy now is an established part of our postoperative management in the clinical situation.

The Fontan operation is performed by us in the last few years routinely with extracardiac conduits with fenestration, which might answer your last question. We think fenestration is important to improve preload reserve in these patients.

\section{Authoritative}

The Journal of Thoracic and Cardiovascular Surgery is the most frequently cited thoracic/cardiovascular surgery journal in the Science Citation Index. An article in JTCVS is cited on average almost twice as often as those in the closest cardiothoracic journal. 